\title{
EXTENDED GLRT DETECTORS OF CORRELATION AND SPHERICITY: THE UNDERSAMPLED REGIME
}

\author{
Xavier Mestre $^{1}$, Pascal Vallet ${ }^{2}$ \\ ${ }^{1}$ Centre Tecnològic de Telecomunicacions de Catalunya, Castelldefels, Barcelona (Spain) \\ ${ }^{2}$ Institut Polytechnique de Bordeaux (ENSEIRB-MATMECA), Bordeux (France)
}

\begin{abstract}
Detecting the presence of one or multiple signals with unknown spatial signature can be addressed by testing the structure of the observation covariance matrix. The problem can be typically formulated as a sphericity test, which checks whether the spatial covariance matrix is proportional to the identity (white noise), or as a correlation test, which checks whether this matrix has a diagonal structure. When the number of samples is higher than the number of antennas, one can address this problem by formulating the generalized likelihood ratio test (GLRT), which basically compares the arithmetic and geometric means of the eigenvalues of the sample covariance/coherence matrix. The GLRT can be trivially extended to the undersampled regime by selecting only the positive sample eigenvalues. This paper investigates the asymptotic behavior of these extended GLRTs by determining the asymptotic law of the associated statistics under both hypotheses. The analysis is asymptotic in both the sample size (number of snapshots) and the observation dimension (number of antennas).
\end{abstract}

Index Terms - GLRT, sphericity test, correlation test, random matrix theory, central limit theorem.

\section{INTRODUCTION}

Multiple detection problems in statistical array signal processing can be formulated as a test on the structure of the spatial covariance matrix of the observations. In radar applications, for example, one may decide that the scenario is free from directional signals if the correlation matrix of the observations is close to the identity matrix, corresponding to the background noise. This test is typically referred to as the "sphericity test", and declares the presence of spatial sources whenever the spatial covariance matrix becomes sufficiently different from a scaled identity [1, p.431]. In some other scenarios, e.g. when the array is not calibrated, the absence of signals results in a generally diagonal spatial covariance matrix. These situations are typically tackled by "correlation

This work was partially supported by the Catalan and Spanish grants 2014 SGR 1567 and TEC2014-59255-C3-1-R and by the European Commission under project Newcom\# (FP7-ICT-318306). tests", which declare the presence of spatial sources whenever the covariance matrix becomes different from a diagonal.

In order to introduce these tests, let us denote by $\mathbf{y}_{n}$ an $M \times 1$ column vector that contains the measurements collected by the $M$ sensors or antennas at the $n$th time instant. We will model these measurements as zero-mean Gaussian random vectors. In order to consider both the real-valued and the complex-valued formulations, we introduce a boolean variable $\varsigma$ such that $\varsigma=1$ when the observations are realvalued and $\varsigma=0$ when they are complex-valued. More formally:

(As1) The set of $M$-dimensional observations $\mathbf{y}_{n}, n=$ $1, \ldots, N$, be independent and identically distributed (i.i.d.) random vectors. If $\varsigma=0, \mathbf{y}_{n} \sim \mathcal{C N}\left(0, \mathbf{R}_{M}\right)$, i.e. complex circularly symmetric, whereas if $\varsigma=1, \mathbf{y}_{n} \sim \mathcal{N}\left(0, \mathbf{R}_{M}\right)$, i.e. real-valued.

The problem of establishing whether $\mathbf{R}_{M}$ is proportional to the identity matrix and can be formulated as a binary hypothesis test, namely

$$
\begin{aligned}
& \mathcal{H}_{0}: \mathbf{R}_{M}=\sigma^{2} \mathbf{I}_{M} \\
& \mathcal{H}_{1}: \mathbf{R}_{M} \neq \sigma^{2} \mathbf{I}_{M}
\end{aligned}
$$

where $\sigma^{2}>0$ is some unknown parameter describing the noise power. In some situations (uncalibrated array receivers, distributed sensor networks, etc.) it becomes more relevant to establish whether the covariance matrix is diagonal, i.e.

$$
\begin{aligned}
& \mathcal{H}_{0}: \mathbf{R}_{M}=\mathbf{D}_{M} \\
& \mathcal{H}_{1}: \mathbf{R}_{M} \neq \mathbf{D}_{M}
\end{aligned}
$$

where $\mathbf{D}_{M}$ is an $M \times M$ unknown positive diagonal matrix that contains the diagonal elements of $\mathbf{R}_{M}$. These two binary hypothesis tests are referred to as the sphericity and the correlation tests respectively in classical multivariate statistical analysis.

One of the most prominent approaches in order to solve these two detection problems consists in reformulating them in terms of the Generalized Likelihood Ratio Test (GLRT). Let us consider the sample correlation matrix $\hat{\mathbf{R}}_{M}$, which is constructed as

$$
\hat{\mathbf{R}}_{M}=\frac{1}{N} \sum_{n=1}^{N} \mathbf{y}_{n} \mathbf{y}_{n}^{H} .
$$


Assuming that the number of samples is higher than the observation dimension $(N \geq M)$, the sphericity GLRT exists, and rejects the null hypothesis for large values of the statistic $[1, \mathrm{p} .431]$

$$
\hat{\eta}_{M}^{\mathrm{sphr}}=\log \left[\frac{1}{M} \operatorname{tr}\left(\hat{\mathbf{R}}_{M}\right)\right]-\frac{1}{M} \log \operatorname{det}\left(\hat{\mathbf{R}}_{M}\right) .
$$

Likewise, let $\hat{\mathbf{D}}_{M}=\hat{\mathbf{R}}_{M} \odot \mathbf{I}_{M}$, where $\odot$ is the Hadamard product, and let $\hat{\mathbf{C}}_{M}$ denote the sample coherence matrix, namely

$$
\hat{\mathbf{C}}_{M}=\hat{\mathbf{D}}_{M}^{-1 / 2} \hat{\mathbf{R}}_{M} \hat{\mathbf{D}}_{M}^{-1 / 2}
$$

where $\hat{\mathbf{D}}_{M}^{1 / 2}$ is the positive square root of $\hat{\mathbf{D}}_{M}$. When $N \geq$ $M$, the correlation GLRT exists, and rejects the null hypothesis for large values of

$$
\hat{\eta}_{M}^{\text {corr }}=\log \left[\frac{1}{M} \operatorname{tr}\left(\hat{\mathbf{C}}_{M}\right)\right]-\frac{1}{M} \log \operatorname{det}\left(\hat{\mathbf{C}}_{M}\right) .
$$

Since the diagonal entries of $\hat{\mathbf{C}}_{M}$ are all equal to one, the first term of the above equation is identically zero. However, we keep this term in order to uniformize the notation with (1).

Unfortunately, in many practical situations the number of samples may be lower than the observation dimension $(N<$ $M)$, which implies that the two above tests do not exist anymore. However, one can trivially extend the above statistics to the case $N<M$ by noticing that they can be formulated as the logarithm of the quotient between the arithmetic and geometric means of the sample eigenvalues. Let $\hat{\lambda}_{1} \leq \ldots \leq \hat{\lambda}_{M}$ denote the eigenvalues of either $\hat{\mathbf{R}}_{M}$ (sphericity test) or $\hat{\mathbf{C}}_{M}$ (correlation test). We may consider an extension of the above GLRT statistics to the undersampled case $(N<M)$ by simply selecting the positive sample eigenvalues of these two matrices, namely

$$
\hat{\eta}_{M}=\log \frac{\frac{1}{M^{+}} \sum_{m=M-M^{+}+1}^{M} \hat{\lambda}_{m}}{\left[\prod_{m=M-M^{++1}}^{M} \hat{\lambda}_{m}\right]^{1 / M^{+}}}
$$

where $M^{+}=\min \{M, N\}$ is the total number of positive eigenvalues. The objective of this paper is the asymptotic characterization of the above statistic under both the null and the alternative hypotheses and assuming that $M$ may be either lower or larger than the sample size $N$. Classical asymptotics, which assume that $M$ stays fixed while $N \rightarrow \infty$, clearly fail to characterize the new undersampled situation. For this reason, we resort to an alternative asymptotic approach that assumes that both $M, N$ grow large at the same rate.

\section{ASYMPTOTIC BEHAVIOR OF THE GLRT}

Our objective is to study the asymptotic behavior of the extended GLRT statistic in (3) when both $M$ and $N$ increase without bound at the same rate. We will basically consider both the cases $M>N$ and $M<N$, and we will obviate the case $M=N$ since it requires more complicated technical tools. We will use the following assumptions:

(As2) The observation dimension $M$ is a function of $N$. Its quotient, denoted by $c_{N}=M / N$, is such that $c_{N} \neq 1$ and $\lim _{N \rightarrow \infty} c_{N}=c, 0<c<\infty, c \neq 1$.

(As3) If $\lambda_{\min }\left(\mathbf{R}_{M}\right)$ and $\lambda_{\max }\left(\mathbf{R}_{M}\right)$ denote the minimum and maximum eigenvalues of the Hermitian matrix $\mathbf{R}_{M}$, $\inf _{M} \lambda_{\min }\left(\mathbf{R}_{M}\right)>0$ and $\sup _{M} \lambda_{\max }\left(\mathbf{R}_{M}\right)<\infty$.

In order to address this problem, we notice that we can generally express (3) in integral form as follows. Consider the function $\hat{m}_{M}(z)$, defined as

$$
\hat{m}_{M}(z)=\frac{1}{M^{+}} \sum_{m=1}^{M} \frac{1}{\hat{\lambda}_{m}-z} .
$$

Then, one can express $\hat{\eta}_{M}$ for both the correlation and sphericity extended GLRT as

$$
\hat{\eta}_{M}=\log \left[\hat{I}_{M}\{z\}\right]-\hat{I}_{M}\{\log z\}
$$

where, given a complex function $f(z)$ that is analytic on the positive real axis, we have denoted

$$
\hat{I}_{M}\{f(z)\}=\frac{1}{2 \pi \mathrm{j}} \oint_{C_{M}^{-}} f(z) \hat{m}_{M}(z) d z
$$

and where $C_{M}^{-}$is a simple negatively (clockwise) oriented contour enclosing all the positive sample eigenvalues and not zero. Using this representation, one can easily address the first order asymptotic behavior of $\hat{\eta}_{M}$ by simply studying the asymptotic behavior of $\hat{m}_{M}(z)$. This analysis is valid regardless of whether $\hat{\eta}_{M}$ is constructed from the eigenvalues of either $\hat{\mathbf{R}}_{M}$ or $\hat{\mathbf{C}}_{M}$.

Let $\mathbf{C}_{M}$ denote the true coherence matrix, namely $\mathbf{C}_{M}=$ $\mathbf{D}_{M}^{-1 / 2} \mathbf{R}_{M} \mathbf{D}_{M}^{-1 / 2}$. Assume that $\mathbf{R}_{M}$ (alternatively $\mathbf{C}_{M}$ ) presents $\bar{M}$ distinct eigenvalues, denoted as $\gamma_{1}<\ldots<\gamma_{\bar{M}}$, and assume that the $m$ th eigenvalue $\gamma_{m}$ has multiplicity $K_{m}$, so that $K_{1}+\cdots+K_{\bar{M}}=M$. It turns out that, for both sample covariance [2] and sample coherence ${ }^{1}$ [3] matrices, the random function $\hat{m}_{M}(z)$ is asymptotically close to a certain deterministic equivalent $\bar{m}_{M}(z)$, in the sense that $\left|\bar{m}_{M}(z)-\hat{m}(z)\right| \rightarrow 0$ almost surely for any $z \in \mathbb{C}^{+} \equiv\{z: \operatorname{Im} z>0\}$. This deterministic equivalent $\bar{m}_{M}(z)$ is defined as

$$
\bar{m}_{M}(z)=\frac{\omega_{M}(z)}{z} \frac{1}{M^{+}} \sum_{m=1}^{M} K_{m} \frac{1}{\gamma_{m}-\omega_{M}(z)}
$$

where $\omega_{M}(z)$ denotes the unique solution in $\mathbb{C}^{+}$to the following equation in $\omega$

$$
z=\omega\left(1-\frac{1}{N} \sum_{m=1}^{M} K_{m} \frac{\gamma_{m}}{\gamma_{m}-\omega}\right) .
$$

\footnotetext{
${ }^{1}$ The results in [3] are presented for the complex-valued case. However, these results can easily be generalized to the real-valued scenario.
} 
Define $\lambda_{\text {sup }}$ and $\lambda_{\text {inf }}$ as the following lower/upper bounds:

$$
\lambda_{\text {inf }}=\inf _{M} \lambda_{\min }\left(\mathbf{R}_{M}\right), \lambda_{\text {sup }}=\sup _{M} \lambda_{\max }\left(\mathbf{R}_{M}\right)
$$

and let $s=\lambda_{\text {sup }} / \lambda_{\text {inf }}>1$. Using the results in [4] it can easily be shown that, with probability one and for sufficiently large $M$, all the eigenvalues of $\hat{\mathbf{R}}_{M}$ and $\hat{\mathbf{C}}_{M}$ are located inside the intervals $\left[(1-\sqrt{c})^{2} \lambda_{\text {inf }},(1+\sqrt{c})^{2} \lambda_{\text {sup }}\right]$ and $\left[(1-\sqrt{c})^{2} s^{-1},(1+\sqrt{c})^{2} s\right]$ respectively. We will generally denote by $\mathcal{S}$ this interval, its actual definition being apparent from the context. The two complex functions $\bar{m}_{M}(z)$ and $\omega_{M}(z)$ can be analytically extended to $\mathbb{C} \backslash(\mathcal{S} \cup\{0\})$. It follows from the dominated convergence theorem that, under (As1)-(As3) and for any complex function $f(z)$ analytic on an open subset containing $\mathcal{S}$, we will have

$$
\hat{I}_{M}\{f(z)\}-\bar{I}_{M}\{f(z)\} \rightarrow 0
$$

where we have defined

$$
\bar{I}_{M}\{f(z)\}=\frac{1}{2 \pi \mathrm{j}} \oint_{\mathcal{C}^{-}} f(z) \bar{m}_{M}(z) d z
$$

with $\mathcal{C}^{-}$denoting a simple negatively oriented contour enclosing $\mathcal{S}$ and not zero (this is always possible, since $c_{N} \neq 1$ by assumption). The immediate consequence of this result in the two considered tests can be formalized as follows.

Theorem 1. Under (As1) - (As3), the extended GLRT statistic for both correlation and sphericity detection defined in (3) is asymptotically close to a deterministic equivalent $\bar{\eta}_{M}$, in the sense that $\left|\hat{\eta}_{M}-\bar{\eta}_{M}\right| \rightarrow 0$ almost surely, where

$$
\bar{\eta}_{M}=\eta_{M}+\frac{M}{M^{+}}+\frac{N-M}{M^{+}} \log \left|1-\frac{M}{N}\right|
$$

if $c<1$ and

$$
\begin{aligned}
\bar{\eta}_{M} & =\eta_{M}+\frac{N}{M^{+}}-\frac{N-M}{M^{+}} \log \left|\omega_{*}\right| \\
& +\frac{1}{M^{+}} \sum_{m=1}^{M} K_{m} \log \left|\frac{\gamma_{m}}{\gamma_{m}-\omega_{*}}\right|
\end{aligned}
$$

when $c>1$, where we have defined

$$
\eta_{M}=\log \left[\frac{1}{M^{+}} \sum_{m=1}^{M} K_{m} \gamma_{m}\right]-\frac{1}{M^{+}} \sum_{m=1}^{M} K_{m} \log \gamma_{m}
$$

and where $\omega_{*} \leq 0$ is the smallest solution to the equation

$$
1=\frac{1}{N} \sum_{m=1}^{M} K_{m} \frac{\gamma_{m}}{\gamma_{m}-\omega_{*}} .
$$

The proof of this results follows directly from (6) and the continuity of the logarithm, which directly implies that $\left|\hat{\eta}_{M}-\bar{\eta}_{M}\right| \rightarrow 0$ almost surely for $\bar{\eta}_{M}$ defined as

$$
\bar{\eta}_{M}=\log \left[\bar{I}_{M}\{z\}\right]-\bar{I}_{M}\{\log z\} .
$$

The only difficult point in the proof of Theorem 1 comes from the computation of the integrals $\bar{I}_{M}\{f(z)\}$ in (7) for $f(z)=z$ and $f(z)=\log z$, so as to show that this $\bar{\eta}_{M}$ corresponds to the one in the statement of the Theorem. In order to solve these integrals, we introduce the change of variable $z \mapsto \omega=\omega_{M}(z)$, see [5] for further details. This change of variable allows to obtain $\bar{I}_{M}\{z\}$ using the classical residue theorem. As for the second term in $\bar{\eta}_{M}$, corresponding to the integral with $f(z)=\log z$, we only need to observe that, by the definition of $\omega_{*}$ we can write (by the $z \mapsto \omega$ change of variable)

$\log z=\log \left[\left(\omega-\omega_{*}\right)\left(1-\frac{1}{N} \sum_{m=1}^{M} \frac{K_{m} \gamma_{m}^{2}}{\left(\gamma_{m}-\omega\right)\left(\gamma_{m}-\omega_{*}\right)}\right)\right]$

Inserting this into the definition of $\bar{I}_{M}\{\log z\}$ and using the techniques developed in [6], we obtain the result in this theorem.

Observe that, contrary to what happens with classical asymptotics, here the GLRT statistic does not converge to $\eta_{M}$ and an asymptotic bias term appears, which critically depends on the quotient $M / N$. This bias term disappears when $M / N \rightarrow 0$, agreeing with the fact that $\hat{\eta}_{M} \rightarrow \eta_{M}$ when $N \rightarrow \infty$ for a fixed $M$. On the other hand, it is interesting to point out that the same expression for $\bar{\eta}_{M}$ in Theorem 1 is valid for both the correlation and the sphericity tests, although one should keep in mind that the eigenvalues $\gamma_{k}$ have a different definition depending on the actual test. In fact, the deterministic equivalent of the extended correlation GLRT accepts further simplifications by using the fact that the diagonal entries of the coherence matrix are all equal to one, so that $\sum_{m=1}^{M} K_{m} \gamma_{m}=M$, and therefore the first term of $\eta_{M}$ becomes equal to $\log \left(M / M^{+}\right)$.

Next, we provide a more interesting result that characterizes the asymptotic fluctuations of $\hat{\eta}_{M}$ around $\bar{\eta}_{M}$ in this asymptotic regime.

\section{A CENTRAL LIMIT THEOREM ON $\hat{\eta}_{M}$}

In this section, we analyze the behavior of the two GLRT in terms of fluctuations around the deterministic equivalents $\bar{\eta}_{M}$. Here, the behavior turns out to be substantially different depending on the actual test (sphericity or correlation). The following result generalizes the results in [7] (by allowing $c>1$ in the sphericity test) and also in [3] (by also including the $c>1$ case and allowing for real-valued observations in the correlation test). From now on we omit the dependence on $M$ in all matrices to simplify the notation.

Theorem 2. Let $\mathcal{L}_{M}$ be a deterministic quantity defined as

$$
\mathcal{L}_{M}=-\log \left|1-\frac{1}{N} \sum_{m=1}^{\bar{M}} \frac{K_{m} \gamma_{m}^{2}}{\left(\gamma_{m}-\omega_{*}\right)^{2}}\right|
$$


and let $\mu_{M}$ and $\sigma_{M}^{2}$ be defined as follows. For the extended sphericity GLRT, we take $\mu_{M}=\varsigma \mathcal{L}_{M} / 2$ and

$$
\begin{aligned}
\sigma_{M}^{2} & =(1+\varsigma)\left[\mathcal{L}_{M}+\left(\frac{1}{M^{+}} \operatorname{tr}[\mathbf{R}]\right)^{-2} \frac{1}{N} \operatorname{tr}\left[\mathbf{R}^{2}\right]\right] \\
& -2(1+\varsigma)\left(\frac{1}{M^{+}} \operatorname{tr}[\mathbf{R}]\right)^{-1} \frac{1}{N} \operatorname{tr}\left[\mathbf{R}^{2} \mathbf{\Psi}\left(\omega_{*}\right)\right]
\end{aligned}
$$

where $\boldsymbol{\Psi}(\omega)=\left(\mathbf{R}-\omega \mathbf{I}_{M}\right)^{-1}$. For the extended correlation GLRT, we take

$$
\begin{aligned}
\mu_{M} & =\frac{\varsigma}{2} \mathcal{L}_{M}-\frac{1+\varsigma}{2} \frac{M}{N}+\frac{\omega_{*}^{2}}{2} \frac{1+\varsigma}{N} \operatorname{tr}\left[\left(\mathbf{C} \odot \Theta\left(\omega_{*}\right)\right)^{2}\right] \\
& -\frac{1+\varsigma}{N} \omega_{*} \operatorname{tr}\left[\mathbf{C} \Theta\left(\omega_{*}\right) \odot \mathbf{C} \Theta\left(\omega_{*}\right)\right]
\end{aligned}
$$

and

$$
\begin{aligned}
\sigma_{M}^{2} & =(1+\varsigma)\left[\mathcal{L}_{M}-\frac{2}{N} \operatorname{tr}\left[\mathbf{C}^{2} \Theta\left(\omega_{*}\right) \odot \mathbf{C} \Theta\left(\omega_{*}\right)\right]\right] \\
& +\frac{1+\varsigma}{N} \operatorname{tr}\left[\left(\mathbf{C}\left(\mathbf{C} \Theta\left(\omega_{*}\right) \odot \mathbf{I}_{M}\right)\right)^{2}\right]
\end{aligned}
$$

where $\Theta(\omega)=\left(\mathbf{C}-\omega \mathbf{I}_{M}\right)^{-1}$. Under $(\mathbf{A s} \mathbf{1})-(\mathbf{A s 3})$,

$$
\sigma_{M}^{-1}\left(M\left(\hat{\eta}_{M}-\bar{\eta}_{M}\right)-\mu_{M}\right) \stackrel{\mathcal{L}}{\longrightarrow} \mathcal{N}(0,1) .
$$

According to the above result, both correlation and sphericity extended GLRT asymptotically fluctuate as Gaussian random variables. This was well known for the case $c<1$ (more samples than sensors) but here we see that the result is also valid for the undersampled scenario ${ }^{2}(c>1)$. Observe also that when $c<1$ we have $\omega_{*}=0$ and this leads to the results presented in [7] and [3] for the sphericity and correlation tests respectively.

Let us next provide a sketch of the proof of Theorem 2 . The basis of the proof relies on the following result. Define complex functions $\mu_{M}(\omega)$ and $\sigma_{M}^{2}\left(\omega_{1}, \omega_{2}\right)$ as follows. For the sample coherence matrix (correlation test), take

$$
\begin{aligned}
\bar{\mu}_{M}(\omega) & =\varsigma \frac{\frac{1}{N} \operatorname{tr}\left[\mathbf{C}^{2} \Theta^{3}(\omega)\right]}{1-\frac{1}{N} \operatorname{tr}\left[\mathbf{C}^{2} \Theta^{2}(\omega)\right]} \\
& +\frac{1+\varsigma}{N} \operatorname{tr}\left[\left(\mathbf{C} \Theta(\omega)-2 \mathbf{C}^{2} \Theta^{2}(\omega)\right) \odot \mathbf{C} \Theta(\omega)\right] \\
& +\omega \frac{1+\varsigma}{N} \operatorname{tr}\left[\left(\mathbf{C} \Theta^{2}(\omega) \odot \mathbf{C}\right)(\Theta(\omega) \odot \mathbf{C})\right] .
\end{aligned}
$$

and

$$
\begin{aligned}
\bar{\sigma}_{M}^{2}\left(\omega_{1}, \omega_{2}\right) & =\frac{1+\varsigma}{\left(\omega_{1}-\omega_{2}\right)^{2}}-\frac{1+\varsigma}{N} \operatorname{tr}\left[\mathbf{C}^{2} \Theta^{2}\left(\omega_{1}\right) \Theta^{2}\left(\omega_{2}\right)\right] \\
& +\frac{1+\varsigma}{N} \operatorname{tr}\left[\mathbf{C} \boldsymbol{\Delta}\left(\omega_{1}\right) \mathbf{C \Delta}\left(\omega_{2}\right)\right]
\end{aligned}
$$

\footnotetext{
${ }^{2}$ One can conjecture that the result will also be valid for the case $c=$ 1 , although the techniques used in our proof are not valid to address this situation.
}

where $\boldsymbol{\Delta}(\omega)=\Theta^{2}(\omega)-\left[\mathbf{C} \Theta^{2}(\omega) \odot \mathbf{I}_{M}\right]$. For the sample correlation matrix (sphericity test), take only the first term in the above two definitions, replacing $\mathbf{C}$ by $\mathbf{R}$ and $\Theta(\omega)$ by $\boldsymbol{\Psi}(\omega)$ respectively. Let $f_{1}(z)$ and $f_{2}(z)$ denote two complex functions that are analytic on the positive real axis, and define the $2 \times 1$ column vector $\mathbf{m}_{M}$ and the $2 \times 2$ matrix $\boldsymbol{\Phi}_{M}$ as

$$
\begin{aligned}
\left\{\mathbf{m}_{M}\right\}_{i} & =\frac{1}{2 \pi \mathrm{j}} \oint_{\mathcal{C}_{\omega}^{-}} F_{i}(\omega) \bar{\mu}_{M}(\omega) d \omega \\
\left\{\boldsymbol{\Phi}_{M}\right\}_{i, j} & =\frac{-1}{4 \pi^{2}} \oint_{\mathcal{C}_{\omega}^{-}} \oint_{\mathcal{C}_{\omega}^{-}} F_{i}\left(\omega_{1}\right) F_{j}\left(\omega_{2}\right) \bar{\sigma}_{M}^{2}\left(\omega_{1}, \omega_{2}\right) d \omega_{1} d \omega_{2}
\end{aligned}
$$

where $\mathcal{C}_{\omega}=\omega_{M}(\mathcal{C})$ and $F_{i}(\omega), i=1,2$, is equal to $f_{i}(z)$ after replacing $z$ by the right hand side of (5). Let $\kappa$ denote a fixed $2 \times 1$ complex deterministic vector and assume that $\left|\operatorname{Re}\left[\kappa^{H} \mathbf{m}_{M}\right]\right|$ is bounded, and that $\kappa^{H} \boldsymbol{\Phi}_{M} \kappa$ is bounded from infinity and away from zero. Consider the column vector

$$
\hat{\mathbf{z}}_{N}=M^{+}\left[\begin{array}{c}
\bar{I}_{M}\left\{f_{1}(z)\right\}-\hat{I}_{M}\left\{f_{1}(z)\right\} \\
\bar{I}_{M}\left\{f_{2}(z)\right\}-\hat{I}_{M}\left\{f_{2}(z)\right\}
\end{array}\right]
$$

Then, under (As1)-(As3),

$$
\frac{\operatorname{Re}\left(\kappa^{H} \hat{\mathbf{z}}_{N}\right)-\operatorname{Re}\left(\kappa^{H} \mathbf{m}_{M}\right)}{\sqrt{\kappa^{H} \mathbf{\Phi}_{M} \kappa}} \stackrel{\mathcal{L}}{\longrightarrow} \mathcal{N}(0,1) .
$$

This result follows directly from [7] for the sample covariance matrix and from [3] (with some modifications) for the sample coherence matrix. Theorem 2 will follow directly from the application of this result to the problem at hand, particularizing $f_{1}(z)=z$ and $f_{2}(z)=\log z$.

Indeed, consider the expression of $\hat{\eta}_{M}$ and $\bar{\eta}_{M}$ in (4)-(8), and observe that we can express (using a first order Taylor approximation of the logarithm)

$$
\begin{aligned}
M^{+}\left[\hat{\eta}_{M}-\bar{\eta}_{M}\right] & =\frac{1}{\bar{I}_{M}\{z\}} M^{+}\left(\hat{I}_{M}\{z\}-\bar{I}_{M}\{z\}\right) \\
& -M^{+}\left(\hat{I}_{M}\{\log z\}-\bar{I}_{M}\{\log z\}\right) \\
& -\frac{1}{M^{+} \delta_{M}^{2}}\left[M^{+}\left(\hat{I}_{M}\{z\}-\bar{I}_{M}\{z\}\right)\right]^{2}
\end{aligned}
$$

where $\delta_{M}$ belongs to the interval between the two quantities $\bar{I}_{M}\{z\}, \hat{I}_{M}\{z\}$. The magnitude of these two quantities is a.s. bounded away from zero for all large $M$, so that by using (9) with $\kappa=[1,0]^{T}$ we can readily see that the third term of the above expression converges in probability to zero and can therefore be obviated. Now, using again (9) with $\kappa=\left[\bar{I}_{M}^{-1}\{z\},-1\right]^{T}$, and applying the integration technique developed in [6], one can check that $\kappa^{H} \mathbf{m}_{M}=\mu_{M}$ and $\kappa^{H} \boldsymbol{\Phi}_{M} \kappa=\sigma_{M}^{2}$, where $\mu_{M}$ and $\sigma_{M}^{2}$ are given by the statement of the theorem. These quantities are bounded as required (we omit the details due to space constraints), so that Theorem 2 follows directly from the convergence in (9). 


\section{NUMERICAL RESULTS}

To illustrate the accuracy of the asymptotic description of the above tests, we consider a radar detection problem where the objective of the tests is to discover the presence of one or multiple Gaussian sources received in spatially white additive background noise. Under the null hypothesis (no signals), the covariance matrix of the observations was identical to the identity matrix, i.e. $\mathbf{R}_{M}=\mathbf{I}_{M}$. Under the alternative hypothesis, we generated a scenario where four different independent complex Gaussian sources are received by a uniform linear array (elements separated half a wavelength) from angles of arrival equal to $[-45,5,15,25]$ degrees with a signal to noise ratio equal to $10 \mathrm{~dB}$. Figures 1 and 2 represent the probability of detection and the probability of false alarm of the extended sphericity and correlation GLRT detectors for different values of the sample size when the total number of antennas was fixed to $M=15$ and $M=30$ antennas respectively. Apart from the empirical probabilities obtained by averaging over $10^{5}$ independent test realizations, we also represent the asymptotic probabilities obtained from the Gaussian distribution in Theorem 2. Observe that there is a perfect match between theoretical and simulated probabilities, for both the undersampled and the oversampled regimes, even for relatively moderate values of $M, N$.
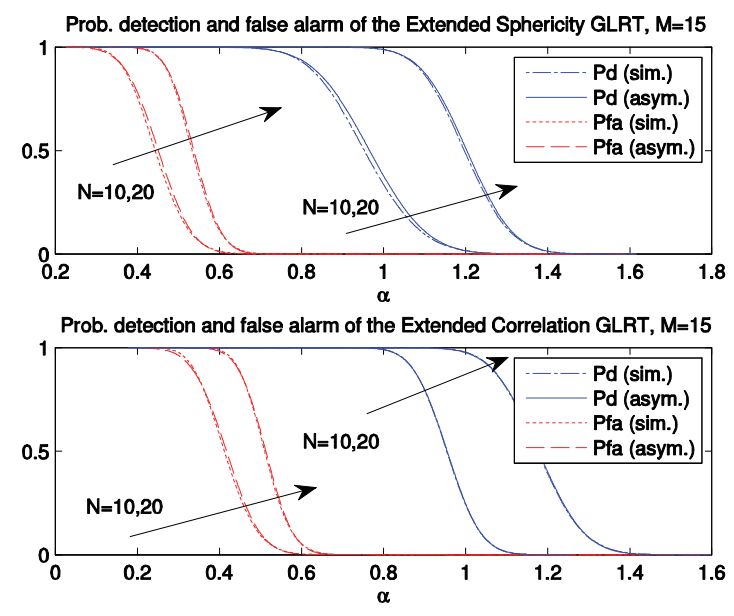

Fig. 1. Empirical and asymptotic probabilities of detection and false alarm as a function of the threshold $(\alpha)$ for $M=15$.

\section{CONCLUSIONS}

This paper has characterized the asymptotic behavior of the extended GLRT for sphericity and correlation, giving special emphasis to the undersampled regime, which had not been investigated before. It has been shown that both extended sphericity and correlation GLRTs asymptotically fluctuate as Gaussian random variables with some specific means
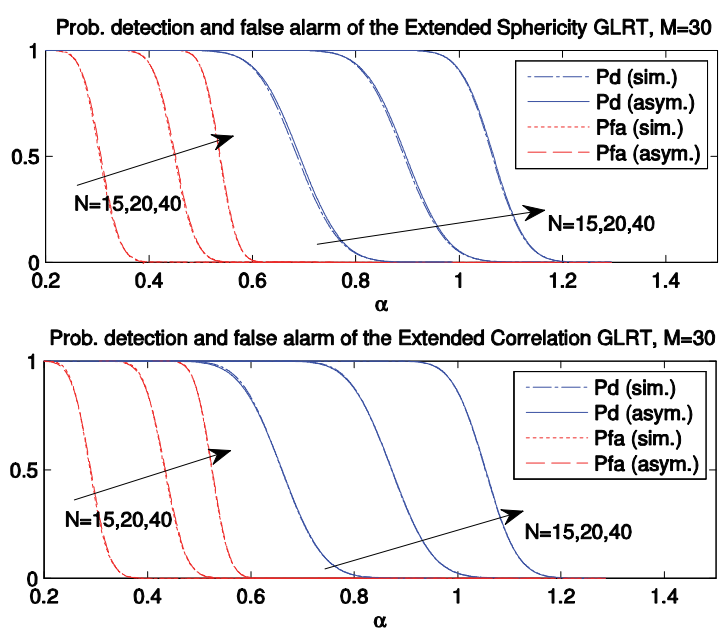

Fig. 2. Empirical and asymptotic probabilities of detection and false alarm as a function of the threshold $(\alpha)$ for $M=30$.

and variances that depend on the true correlation and coherence matrices. Simulations illustrate the accuracy of this asymptotic description, even for relatively low values of the sample size and observation dimension.

\section{REFERENCES}

[1] T.W. Anderson, An Introduction to Multivariate Statistical Analysis, John Wiley and Sons, Stanford, 1984.

[2] J.W. Silverstein, "Strong convergence of the empirical distribution of eigenvalues of large dimensional random matrices," J. Multiv. Anal., vol. 5, pp. 331-339, 1995.

[3] X. Mestre, P. Vallet, and W. Hachem, "Asymptotic analysis of liinear spectral statistics of the sample coherence matrix," in Proceedings of the IEEE Int. Conf. Acoustics, Speech and Sig. Proc. (ICASSP), Adelaide (Australia), April 2015

[4] Z.D. Bai and J.W. Silverstein, "No eigenvalues outside the support of the limiting spectral distribution of large dimensional sample covariance matrices," Ann. Prob., vol. 26, pp. 316-345, 1998.

[5] X. Mestre, "On the asymptotic behavior of the sample estimates of eigenvalues and eigenvectors of covariance matrices," IEEE Trans. Sig. Proc., vol. 56, no. 11, pp. 5353-5368, Nov. 2008.

[6] X. Mestre and P. Vallet, "Improved estimation of the logarithm of the covariance matrix," in Proceedings of the IEEE SAM Workshop (SAM), June 2012, pp. 377-380.

[7] Z.D. Bai and J.W. Silverstein, "CLT for linear spectral statistics of large-dimensional sample covariance matrices," Ann. Prob., vol. 32, no. 1A, pp. 553-605, 2004. 